\title{
Study on the expression of PAK4 and P54 protein in breast cancer
}

Yanqing $\mathrm{Bi}^{1+}$, Mengzi Tian ${ }^{1+}$, Jinghong $\mathrm{Le}^{2}$, Linlin Wang ${ }^{3}$, Xiaofang Liư ${ }^{1}$, Jianhua $\mathrm{Qu}^{1}$ and Min Hao ${ }^{1^{*}}$

\begin{abstract}
Background: Previous evidence have demonstrated that p21-activated kinase PAK4 was correlated with breast cancer. The aim of this paper is to study the expression and interaction of p21-activated kinase (pAK)-4 and P54 protein in breast cancer.

Methods: A total of 80 patients were enrolled in our study (breast fibroma $n=20$, breast noninvasive cancer $n=20$, early breast invasive cancer $n=20$, and advanced breast invasive cancer). The expression of PAK4 was detected by immunohistochemical S-P method, and the relationship between them and the different pathological characteristics were compared. The subcellular localization of P54 and PAK4 in vitro was observed by immunofluorescence assay.

Results: The expression of both PAK4 and P54 in breast cancer was much higher than that in breast fibroma. Meanwhile, we found that both PAK4 and P54 increased gradually as breast cancer progressed (advanced invasive > early invasive > noninvasive). The positive staining of P54 were mainly located in the cytoplasm, especially around the nucleus. There was no significant stained region in the cell matrix. The P54 localization in the cytoplasm was verified by confocal experiment, and the PAK4 was co-localized.
\end{abstract}

Conclusions: PAK4 and P54 proteins may be used as molecular markers for diagnosis and treatment of breast cancer.

Keywords: PAK4, P54, Breast cancer

\section{Background}

Breast cancer ranks the first among women malignant tumors, accounting for 7 to $10 \%$ of the whole body cancer. Peripheral blood tumor marker detection is an important measure for early diagnosis of breast cancer. CA125, CEA, and TPS are the present common serum tumor markers. HER-2/Neu, estrogen receptor (ER), progesterone receptor (PR), epidermal growth factor receptor (EGFR), vascular endothelial growth factor receptor (VEGFR), and vascular endothelial growth factor (VEGF) are detected by pathological specimens. The positive rate and positive predictive value are not that satisfactory and are often used as reference index for clinical diagnosis and treatment. Along with advancement of scientific research, the pathways for phosphorylation/de-phosphorylation of proteins are found to be important for signal transduction, and their balance has

\footnotetext{
* Correspondence: hqa814@163.com

${ }^{\dagger}$ Equal contributors

${ }^{1}$ Department of Breast and Thyroid Surgery, Dongying People's Hospital, No.

317, Nanyi Road, Dongying 257091, Shandong, China

Full list of author information is available at the end of the article
}

a crucial role in the cell's normal signal transduction and tumor occurrence or development. There have been some studies that p21-activated kinase (pAK)-4 has a high expression in breast cancer and ovarian cancer [1-3]. The purpose of our study was to explore the expression of PAK4 and P54 in breast cancer and the correlation of these proteins with pathological stages of breast cancer.

\section{Methods}

Clinical specimens

A total of 80 patients from April 2013 to April 2015 were enrolled in our study (breast fibroma $n=20$, breast noninvasive cancer $n=20$, early breast invasive cancer $n=20$, and advanced breast invasive cancer).

The ages of patients with breast fibroma ranged from 30 to 72 years with a mean value of $44.5 \pm 16.3$ years. And the ages of patients with breast noninvasive cancer ranged from 29 to 66 years with a mean value of $42.5 \pm$ 10.3 years. Among them, there were five ER-positive cases, four PR-positive cases, and three double-positive cases. The ages of patients with early breast invasive 
cancer ranged from 32 to 73 years with a mean value of $45.6 \pm 14.2$ years. Among them, there were four ERpositive cases, five PR-positive cases, and five doublepositive cases. The ages of patients with advanced breast invasive cancer ranged from 46 to 77 years with a mean value of $52.3 \pm 16.7$ years. Among them, there were six ER-positive cases, four PR-positive cases, and four double-positive cases.

All the patients were treated for the first time without drug treatment, surgical resection, chemotherapy history, severe heart, liver, kidney, other organ dysfunction, or other malignant tumors, etc. For the study, we obtained the consent of the patients and their families, and this study was approved by the ethics committee of our hospital. For the same tissue, specimens were obtained from three different loci and completed by the same experienced expert, and the specimens were all fixed by $10 \%$ formalin and embedded in paraffin with 4$\mu \mathrm{m}$ continuous slicing.

\section{Immunohistochemistry S-P method \\ Main reagents and equipment}

For this study, we used the following: rabbit IgG (Nanjing Kaiji Company) and peroxidase-labeled goat anti-rabbit IgG (Shenzhen Jinmei Biological Co., Ltd.), antibody dilutions (Wuhan Boshide Biotechnology Company), constitutive rafter acid salt buffer liquid powder (Nanjing Kaiji Company), and neutral gum (Shanghai Specimen Model Factory). Paraffin sections made from a tissue-slicing machine (Leica, Germany) were dewaxed in water; $3 \% \mathrm{H}_{2} \mathrm{O}_{2}$ was incubated at room temperature for $5 \sim 10 \mathrm{~min}$, to eliminate endogenous peroxidase activity. The S-P immunohistochemical reagent kit was provided by Fujian Maixin Biotechnology Company.

\section{Procedure}

The sections on the slides were rinsed three times in water and PBS, to keep the antigen intact (Shanghai Fengxian Shenxin Chemical Factory). After rinsing with PBS three times, goat serum (Guangzhou Jietewei Company) was flooded on sections for $20 \mathrm{~min}$ at room temperature. Subsequently, the first $50 \mu \mathrm{l}$ of rabbit antihuman PAK4 polyclonal antibody (1: 200 dilution) (Germany BioGenes $\mathrm{GmbH}$ ) was added to the sections and incubated at $37{ }^{\circ} \mathrm{C}$ for $1 \sim 2 \mathrm{~h}$, followed by incubation at $4{ }^{\circ} \mathrm{C}$ overnight. The next day, the slides were washed with $\mathrm{PBS}$ at $37^{\circ} \mathrm{C}$ for $45 \mathrm{~min}$, rinsed three times with PBS. Next, biotin-labeled sheep anti-rabbit IgG antibody was added to sections at $37{ }^{\circ} \mathrm{C}$ and incubated for 10 to $30 \mathrm{~min}$. It was followed by three times PBS washing, then HRP streptavidin solution was added and the slides were incubated at $37{ }^{\circ} \mathrm{C}$ for 10 to $30 \mathrm{~min}$. The slides were rinsed with $\mathrm{PBS}$ three times, and $\mathrm{DAB}$ solution (Guangzhou Jietewei Company) was added for $5 \sim 10 \mathrm{~min}$, followed by PBS wash for $10 \mathrm{~min}$. Finally, the sections were counterstained with hematoxylin staining for $2 \mathrm{~min}$, hydrochloric acid alcohol was differentiated, and ammonia turned back to blue. In the next step, the sections were washed with tap water for 10 15 min, dehydrated and transparent, and mounted for microscopic examination (OLYMPUS BX-4O optical microscope, Olympus, Japan).

\section{Scoring for stained sections}

For interpretation of results, the Standard of Campo was followed (3). No color was given 0 points, weak color 1 point, moderate color 2 points, and strong color 3 points. Stained cells accounted for the total number of cells were less than $5 \%$ for 0 points, $5 \sim 25 \%$ for 1 point, $26 \sim 50 \%$ for 2 points, and more than $51 \%$ for 3 points. Finally, the results were determined by the total score of the percentage of positive cells and the staining intensity score: 0 points for negative, 1 to 2 points for weak positive, 3 to 4 points for medium positive, and more than 5 points for the strong positive. The results of the test were double blind-determined by two pathologists based on the same criteria. The different results were double blinddetermined by another pathologist, and the mean value of the three persons was included in the statistics.

\section{Immunofluorescence}

The cells were placed on a 12-hole cell culture plate, until the cells adhere to start expressing P54 and PAK4 protein plasmid; a double free DMEM was added to incubate for $4 \mathrm{~h}$, which was later replaced with a DMEM culture medium, and the cells were cultured for $24 \mathrm{~h}$, to guarantee outward plasmid normal protein expression. After $24 \mathrm{~h}$, the culture medium was discarded and washed three times with PBS. The PBS was discarded, and the cells were fixed with $700 \mu \mathrm{l}$ of $4 \%$ paraform for $10 \mathrm{~min}$. After three times washing with PBS, $0.1 \%$ Triton-X-100 was added to permeabilize the cell at room

Table 1 Expression of PAK4 in different breast tissues

\begin{tabular}{llllllll}
\hline Group & No. of cases & Negative & Weakly positive & Moderately positive & Strong positive & Kruskal-Wallis & $p$ value \\
\hline Normal tissue & 20 & 15 & 5 & 0 & 0 & 15.623 & $<0.001$ \\
Fibroma & 20 & 10 & 7 & 3 & 4 & 2 & \\
Metastatic tumor tissue & 20 & 6 & 8 & 4 & 9 & \\
Breast cancer & 20 & 1 & 2 & 8 & & \\
\hline
\end{tabular}




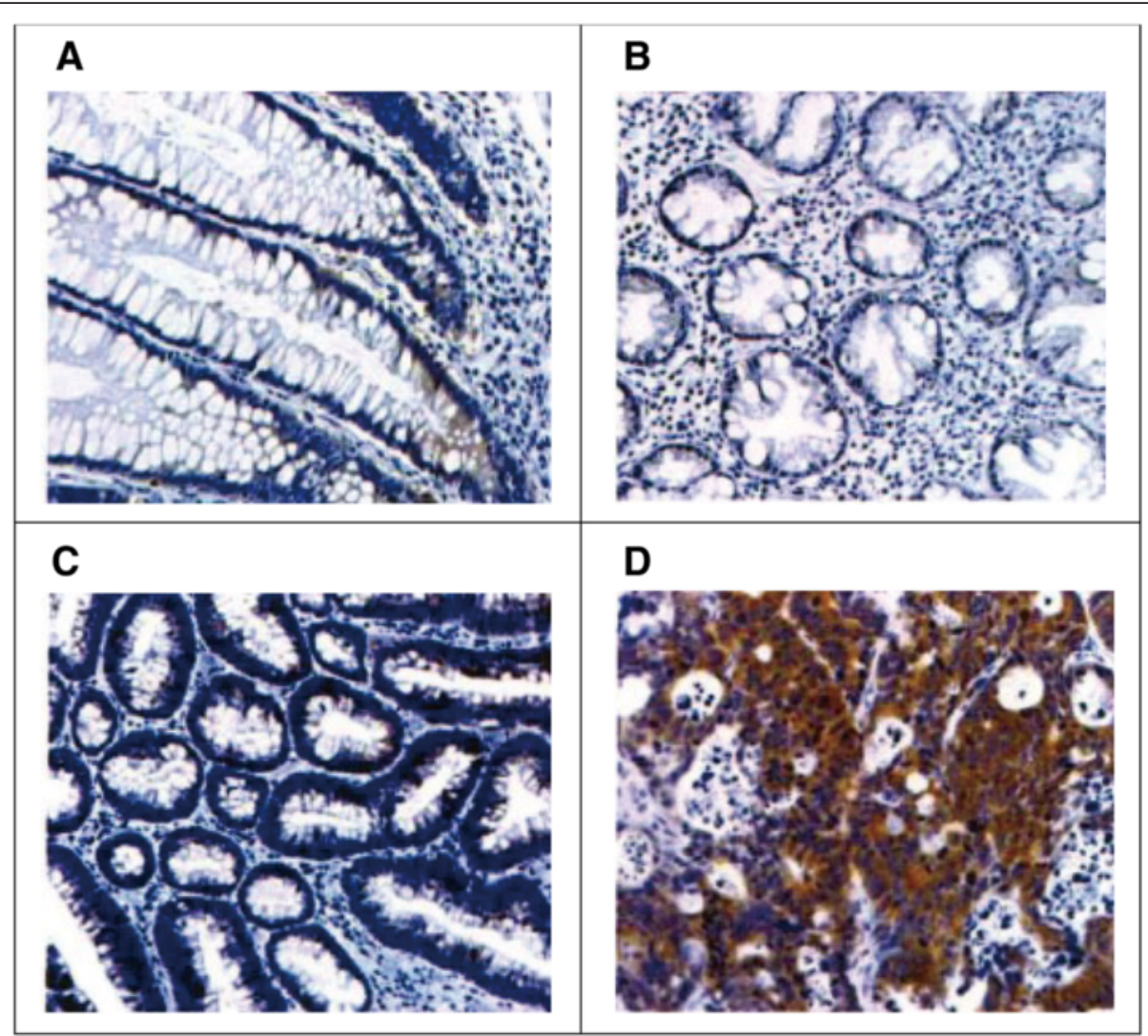

Fig. 1 Expression of PAK4 protein in different breast tissues detected by immunohistochemistry. a Normal tissue. b Fibroma. c Metastatic tumor tissue. $\mathbf{d}$ Breast cancer

temperature for $10 \mathrm{~min}$. Next, again the slides were rinsed with PBS three times, followed by addition of $1 \%$ sheep serum at room temperature for $1 \mathrm{~h}$. Subsequently, the slides were incubated with the anti-PAK4 (1:2000) and anti-myc (1:2000) antibody at room temperature for $1 \mathrm{~h}$ and washed with PBS three times. Subsequently, Alexa-546 red mark goat anti-mouse antibody (1:5000) was added to incubate for $1 \mathrm{~h}$ in dark, followed by washing with PBS three times. Topro-3 nuclear staining was performed for $30 \mathrm{~min}$ and later washed with PBS three times. Finally, $50 \%$ glycerol was added to the slides for mounting, to observe the subcellular localization of proteins in confocal laser scanning microscopy.

\section{Statistical analysis}

SPSS20.0 software (SPSS Chicago, Ill) was used for the statistical analysis. All quantitative data were expressed as mean \pm standard deviation. Comparison between groups was done using one-way ANOVA test followed by a post hoc test (LSD). Percentage (\%) was used to express the enumeration data, and a chi-squared test was used for data analysis. The nonparametric total rank of independent samples of grade data was used to test. $p$ values $<0.05$ were considered statistically significant

\section{Results}

Expression of PAK4 protein in different breast tissues Expression of PAK4 in breast cancer was much higher than that in breast fibroma, and we found that PAK4 increased gradually as breast cancer progressed (advanced invasive $>$ early invasive $>$ noninvasive). The positive products were mainly located in the cytoplasm, especially obvious around the nucleus, and there was no

Table 2 Expression of PAK4 in different pathological types

\begin{tabular}{llllllll}
\hline Pathological type & No. of cases & Negative & Weakly positive & Moderately positive & Strong positive & Kruskal-Wallis & $p$ value \\
\hline Noninvasive carcinoma & 4 & 1 & 1 & 1 & 1 & 12.432 \\
Early invasive carcinoma & 6 & 0 & 1 & 3 & 2 & $<0.001$ \\
Advanced invasive carcinoma & 10 & 0 & 0 & 4 & 6 & \\
\hline
\end{tabular}



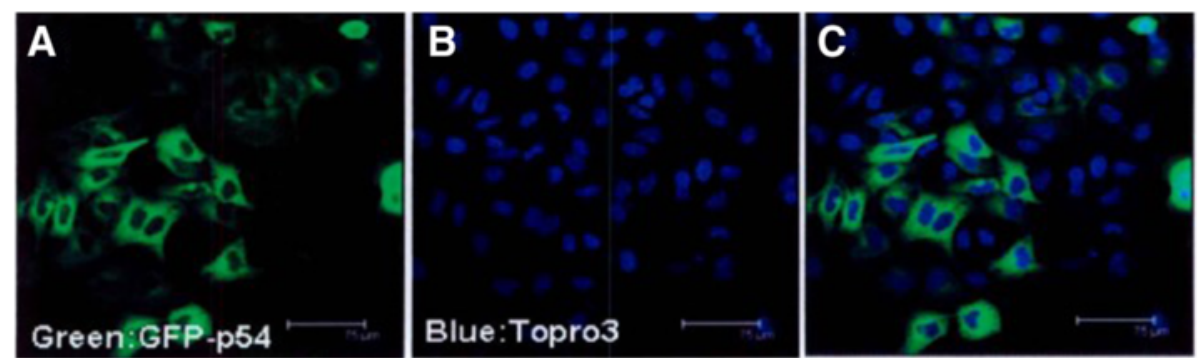

Fig. 2 Subcellular localization of GFP-P54 protein. a Green GFP-p54. b Blue Topro3. c Merged

significant dyeing in the cell matrix $(p<0.05)$ (Table 1 , Fig. 1).

Expression of PAK4 protein in different pathological types Expression of P54 in breast cancer was much higher than that in breast fibroma, and we found that P54 increased gradually as breast cancer progressed (advanced invasive $>$ early invasive $>$ noninvasive). The differences were all statistically significant $(p<0.05)$ (Table 2$)$.

\section{Cellular localization of P54 and PAK4 proteins}

P54 localization in the cytoplasm was verified by confocal experiment, and the PAK4 was co-localized as shown in Figs. 2 and 3. Expression of both PAK4 and P54 in breast cancer was much higher than that in breast fibroma and increased gradually as breast cancer progressed (advanced invasive $32.6 \pm 8.2 \%>$ early invasive $12.4 \pm 3.5 \%>$ noninvasive $2.0 \pm 0.5 \%>$ breast fibroma $0.2 \pm 0.1 \% ; F=20.325, p<0.001)$.

\section{Discussion}

PAK, P21 small GTP-activated kinase, is a serine/threonine protein kinase family. The molecular weight of $21 \mathrm{kD}$, small GTP enzyme of Rho family, Cdc42, and Racl are the upstream molecules of PAKS, in the molecular structure; the end of $\mathrm{N}$ PAK is the regulation region of the kinase; the end of $\mathrm{C}$ is the substrate binding region with kinase activity [4]. PAK4 is a member of the PAK family, which is closely related to human tumor. It plays an important role in the repair of the cytoskeleton, the life of the normal cells, the positive regulation of some normal physiological metabolism, etc. [5-9]. In our study, expression of PAK4 in breast cancer was much higher than that in breast fibroma and increased gradually as breast cancer progressed (advanced invasive $>$ early invasive $>$ noninvasive). The positive products were mainly located in the cytoplasm, around the nucleus, as there was no significant staining in the cell matrix. The positive rate of PAK4 expression in noninvasive carcinoma, early invasive carcinoma, and advanced invasive carcinoma increased gradually, and the differences were all statistically significant. It shows that PAK4 may be used as a sensitive indicator and has important significance in judging the malignant degree and pathological types of breast cancer.

There is some research by constructing PAK4 eukaryotic expression vector and PAK4 ShRNA eukaryotic expression vector, transfecting breast cancer MDA-MB231 cells, which showed that after up-regulation of PAK4 expression in breast cancer cells, it can significantly promote breast cancer cell proliferation; inhibit apoptosis; increase cell adhesion, movement, invasion, tumorigenesis, and other biological activity; and vice versa [10]. The normal mammary gland epithelium has active cell proliferation and cell apoptosis; in normal circumstances, there is a dynamic balance between cell proliferation and cell apoptosis, which is the main mechanism of breast mucosal removal of damaged cells $[11,12]$. PAKs family plays an important role in the process of cell repair. Studies have shown that PAK4 is regulated by growth factors [13].
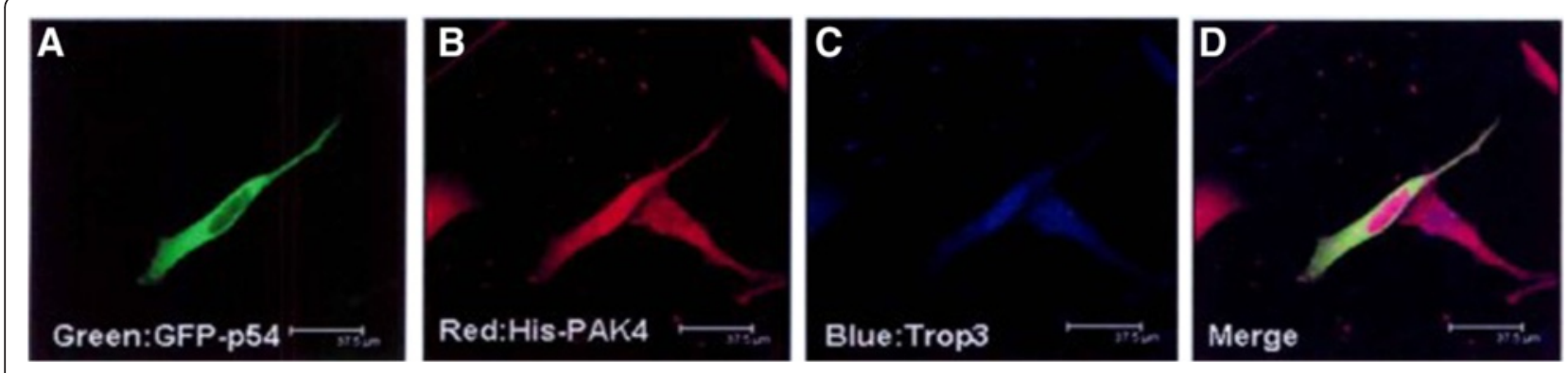

Fig. 3 Cellular co-localization of GFP-P54 protein and PAK4 protein. a Green GFP-p54. b Red His-PAK4. c Blue Topro3. d Merged 
P54 protein is a kind of neuron-specific protein, and it is an effective microtubule instability factor and membraneassociated protein. P54 protein has a certain relationship with tumor, and P54 provides a new basis for the further improvement of PAK4 tumor signaling pathway $[14,15]$. P54 as a member of the family has a structure of three functional regions: the membrane-binding region of the $\mathrm{N}$ end, the central regulatory region, and the coiled coil region of the $C$ end. In the center of regulation of phosphorylation sites, there are phosphorylation sites of MAP and PKA kinase, and these phosphorylation sites can regulate microtubule depolymerization $[16,17]$.

Our study showed that the expression of P54 in breast cancer was much higher than that in breast fibroma and increased gradually as breast cancer progressed (advanced invasive $>$ early invasive $>$ noninvasive). We also found that P54 is located in the cytoplasm with PAK4 co-located. Two hybrid experiments have been shown through yeast, screen-interacting protein P54 and PAK4, and they were transferred to the AH109 yeast strain for yeast verification [18]. The results showed that both could interact; GST pull-down assay results further confirmed the interaction in vitro, and immune precipitation experiment verified the interaction in vivo. In summary, AK4 and P54 proteins may be used as molecular markers for diagnosis and treatment of breast cancer.

\section{Conclusions}

PAK4 expression in breast cancer is adjacent normal tissues, breast fibroma, and breast cancer metastasis tissues, and breast cancer increased gradually. The positive staining was mainly located in the cytoplasm, especially more obvious around the nucleus. Besides, the positive rate of PAK4 expression in noninvasive carcinoma, early invasive carcinoma, and advanced invasive carcinoma also increased gradually. The P54 localization in the cytoplasm and the PAK4 was co-localized, so that PAK4 and P54 proteins may be used as molecular markers for diagnosis and treatment of breast cancer.

\section{Acknowledgements}

The authors thank Dr. Lin-Lin Zhang for her friendship and inspiration to venture into the mitochondrial field and the authors also thank Dr. Hao Wu for his assistance in manuscript preparation.

\section{Authors' contribution}

YB observed the subcellular localization of P54 and PAK4 in vitro by immunofluorescence assay. MT proposed experimental hypothesis and compared the relationship and the different pathological characteristics between PAK4 and P54 protein. JL designed the experimental program and collected the clinical specimens. LW scored for the stained sections, recorded the data, and made the conclusion. XL analyzed the data and wrote the manuscript. JQ detected the expression of PAK4 by immunohistochemical S-P method and recorded the data. $\mathrm{MH}$ analyzed the data, gathered relevant information about the experiment, and found out the problem. All authors read and approved the final manuscript.

\section{Author details}

${ }^{1}$ Department of Breast and Thyroid Surgery, Dongying People's Hospital, No. 317, Nanyi Road, Dongying 257091, Shandong, China. 'Department of General Surgery, Guangrao People's Hospital, Dongying, Shandong, China. ${ }^{3}$ Department of Pathology, Dongying People's Hospital, Dongying, Shandong, China.

Received: 30 December 2015 Accepted: 9 June 2016

Published online: 13 June 2016

References

1. Faure S, Cau J, de Santa BP, Bigou S, Ge Q, Delsert C, Morin N. Xenopus P21-activated kinase 4 regulates blastomeres, adhesive properties during convergent extension movements. Dev Biol. 2005;277:472-92.

2. Kaur R, Liu X, Gjoerup O, Zhang A, Yuan X, Balk SP, Schneider MC, Lu ML. Activation of P21-activated kinase 4 by MAP kinase 6 and p38 MAP kinase. JBC. 2005;280:3323-30.

3. Wong LE, Chen N, Karantza V, Minden A. The Pak4 protein kinase is required for oncogenic transformation of MDA-MB-231 breast cancer cells. Oncogenesis. 2013;2:e50.

4. Wang RA, Zhang H, Balasenthil S, Medina D, Kumar R. PAK 4 hyperactivation is sufficient for mammary gland tumor formation. On-cogene. 2006:25:2931-6.

5. Yang Z, Rayala S, Nguyen D, Vadlamudi RK, Chen S, Kumar R. Pak 4 phosphorylation of snail, a master regulator of epithelial-to-mesen chime transition, modulates snail's subcellular localization and functions. Cancer Res. 2005;65:3179-84.

6. Dart AE, Box GM, Court W, Gale ME, Brown JP, Pinder SE, Eccles SA, Wells CM. PAK4 promotes kinase-independent stabilization of RhoU to modulate cell adhesion. J Cell Biol. 2015;211:863-79.

7. Selamat W, Tay PL, Baskaran Y, Manser E. The cdc42 effector kinase PAK4 localizes to cell-cell junctions and contributes to establishing cell polarity. PLoS One. 2015;10:e129634.

8. Minden A. The PAK4 protein kinase in breast cancer. ISRN Oncol. 2012;2012:694201.

9. Liu Y, Chen N, Cui X, Zheng X, Deng L, Price S, Karantza V, Minden A. The protein kinase PAK4 disrupts mammary acinar architecture and promotes mammary tumorigenesis. Oncogene. 2010;29:5883-94.

10. Singh RR, Song C, Yang Z, Kumar R. Nuclear localization and chromatin targets of P21-activated kinase 4. J Biol Chem. 2005;280:18130-7.

11. Ong CC, Gierke S, Pitt C, Sagolla M, Cheng CK, Zhou W, Jubb AM, Strickland L, Schmidt M, Duron SG, Campbell DA, Zheng W, Dehdashti S, Shen M, Yang N, Behnke ML, Huang W, MCKew JC, Chernoff J, Forrest WF, Haverty PM, Chin SF, Rakha EA, Green AR, Ellis IO, Caldas C, O'Brien T, Friedman LS, Koeppen H, Rudolph J, Hoeflich KP. Small molecule inhibition of group I p21-activated kinases in breast cancer induces apoptosis and potentiates the activity of microtubule stabilizing agents. Breast Cancer Res. 2015;17:59.

12. Radu M, Semenova G, Kosoff $R$, Chernoff J. PAK signalling during the development and progression of cancer. Nat Rev Cancer. 2014;14:13-25.

13. Rudolph J, Crawford JJ, Hoeflich KP, Wang W. Inhibitors of p21-activated kinases (PAKs). J Med Chem. 2015;58:111-29.

14. Stengel KR, Zheng Y. Essential role of Cdc42 in Ras-induced transformation revealed by gene targeting. PLoS One. 2012;7(6):e37317.

15. Gnad F, Young A, Zhou W, Lyle K, Ong CC, Stokes MP, Silva JC, Belvin M, Friedman LS, Koeppen H, Minden A, Hoeflich KP. Systems-wide analysis of K-Ras, Cdc42, and PAK4 signaling by quantitative phosphoproteomics. Mol Cell Proteomics. 2013;12:2070-80.

16. Zhu Z, Zhao X, Zhao L, Yang H, Liu L, Li J, Wu J, Yang F, Huang G, Liu J. p54nrb/NONO regulates lipid metabolism and breast cancer growth through SREBP-1A. Oncogene 2015 [Epub ahead of print]

17. Schiffner S, Zimara N, Schmid R, Bosserhoff AK. p54nrb is a new regulator of progression of malignant melanoma. Carcinogenesis. 2011;32:1176-82.

18. Pavao M, Huang YH, Hafer $\amalg$, Moreland RB, Traish AM. Immunodetection of nmt55/p54nrb isoforms in human breast cancer. BMC Cancer. 2001;1:15. 